\title{
Detecting Sub Bandgap Energies in CIGS with Electron Energy-Loss Spectroscopy
}

\author{
Julia I. Deitz, ${ }^{1}$ Pran K. Paul, ${ }^{2}$ Shankar Karki, ${ }^{3}$ Sylvain Marsillac, ${ }^{3}$ Aaron R. Arehart, ${ }^{2}$ Tyler J. \\ Grassman, ${ }^{1,2}$ and David W. McComb ${ }^{1}$ \\ ${ }^{1}$ Dept. of Materials Science \& Engineering, The Ohio State University, Columbus, OH, 43210, USA. \\ ${ }^{2}$ Dept. of Electrical \& Computer Engineering, The Ohio State University, Columbus, OH, 43210, USA. \\ ${ }^{3}$ Dept. of Electrical \& Computer Engineering, Old Dominion University, Norfolk, VA, 23529, USA.
}

The ultimate performance of semiconductor devices relies on the ability to characterize their structural, chemical, and electronic properties on the nanoscale. Information on defect states, bandgaps, and other electronic transitions throughout nanoscale features can provide vital feedback into the understanding of fundamental behaviors of such materials. For example, in CuInGaSe 2 (CIGS) photovoltaic (PV) devices, electrically active defects limit the ability to achieve the Shockley-Queisser theoretical performance of $32 \%$. Therefore, it is of utmost importance to be able to correlate these defect states with chemical and physical structure to allow for better engineering and efficiency of devices. In this contribution, we present on the detection of sub bandgap energy peaks using electron energy-loss spectroscopy (EELS) in CIGS which correlate with defect energy levels detected via scanning deep level transient spectroscopy (DLTS), strongly suggesting that EELS can be used to detect defect levels in semiconductor materials.

Scanning DLTS and EELS are techniques that can provide high-resolution, spatially-resolved electronic structure information. Scanning-DLTS is a scanning probe microscopy based technique that was recently developed by the authors to achieve nm-scale spatially-resolved defect spectroscopy [1]. It enables mapping of energy-resolved trap concentrations across sample surfaces (with some additional degree of depth sensitivity). This nondestructive method can be paired with local structural and chemical analysis, such as available via electron microscopy analytical methods, to identify the physical sources of defects. EELS can provide higher spatially resolved electronic structure information and electronic structure information below the surface of the sample. However, historical limitations in EELS, such as high energy resolution and Čerenkov radiation have limited the ability to study such information in semiconductors. Recent progress in monochromation for scanning transmission electron microscopy based EELS and extensive research in conditions for limiting Čerenkov radiation in semiconductors currently position EELS to be a technique suitable for detecting sub-bandgap peaks [2].

Scanning DLTS was performed on a CIGS sample as displayed in Figure 1. Figure 1(a) presents a conventional DLTS spectrum where one majority carrier (hole) trap is observed at $\sim 265 \mathrm{~K}$. The activation energy of the trap is $E_{V}+0.43 \mathrm{eV}$, with a capture cross-section of $1.2 \times 10-18 \mathrm{~cm}^{2}$, as extracted via an Arrhenius analysis (Fig. 1 inset). Figure 1(b) presents representative AFM and scanning-DLTS data (at $298 \mathrm{~K}$ ) from the same CIGS surface region. From the scanning-DLTS map, the $\mathrm{E}_{V}+0.43 \mathrm{eV}$ trap is found to be highly concentrated within a specific intergrain region. Figure 2 presents hyperspectral imaging STEM-EELS analysis performed on a CdS-coated CIGS sample prepared under the same conditions. Measurements were performed at $60 \mathrm{kV}$ with a collection angle of $22 \mathrm{mrad}$ to limit the effect of Čerenkov radiation. Figure 2(a) is an EELS zero-loss intensity map of the CIGS region while figure 2(b) presents a valence EELS spectrum, averaged over the region within the white box to improve the signal-to-noise ratio. This spectrum has been further deconvoluted by subtracting the zeroloss signal through the use of the reflected tail method [3]. The inelastic signal after ZLP extraction reveals a peak at $\sim 0.42 \mathrm{eV}$. Of particular interest is the fact that this energy level is consistent with the $\mathrm{E}_{\mathrm{V}}+0.43 \mathrm{eV}$ trap level observed via DLTS. The white area highlighted in Fig. 2(a), within an intergrain 
region and roughly $25-50 \mathrm{~nm}$ below the CIGS/CdS interface, is the only area within the entire $\sim 2 \mu \mathrm{m} \times$ $4 \mu \mathrm{m}$ specimen that exhibited such a strong inelastic signal, consistent with the strong localization observed via scanning-DLTS. Nonetheless, fainter intensities of the $0.42 \mathrm{eV}$ peak/shoulder $(\sim 20 \%$ the intensity of same peak in highlighted area) were observed in other regions of the specimen, which is also consistent with the low concentrations observed along the grain boundary line via scanning-DLTS.

Scanning-DLTS measurements on CIGS have revealed a trap state with an energy of $E_{V}+0.43 \mathrm{eV}$ which was found to be strongly localized within specific grain boundaries. Similar samples grown via the same method (and in the same apparatus) were analyzed using STEM-EELS. An energy-loss peak at $\sim 0.42 \mathrm{eV}$ was observed, consistent with the DLTS results. This signal was also found to be strongly localized within only a small region of the specimen, with weak observations in a few other spots, consistent with scanning-DLTS. These results indicate the potential of high-resolution valence EELS for detection of electronic defect levels, guided by scanning-DLTS, enabling detailed correlative chemical and structural characterization on the nano (and potentially atomic) scale.

[1] P.K. Paul et al., IEEE J. Photovolt., 5, 1482-1486, (2015).

[2] M. Stoger-Pollach, H. Franco, P. Schattschneider, S. Lazar, B. Schaffer, W. Grogger and H. W.

Zandbergen, Micron, 37, 396 (2006).

[3] R. F. Egerton, Electron energy-loss spectroscopy in the electron microscope, 2nd Edition, Plenum press: New York (1996).
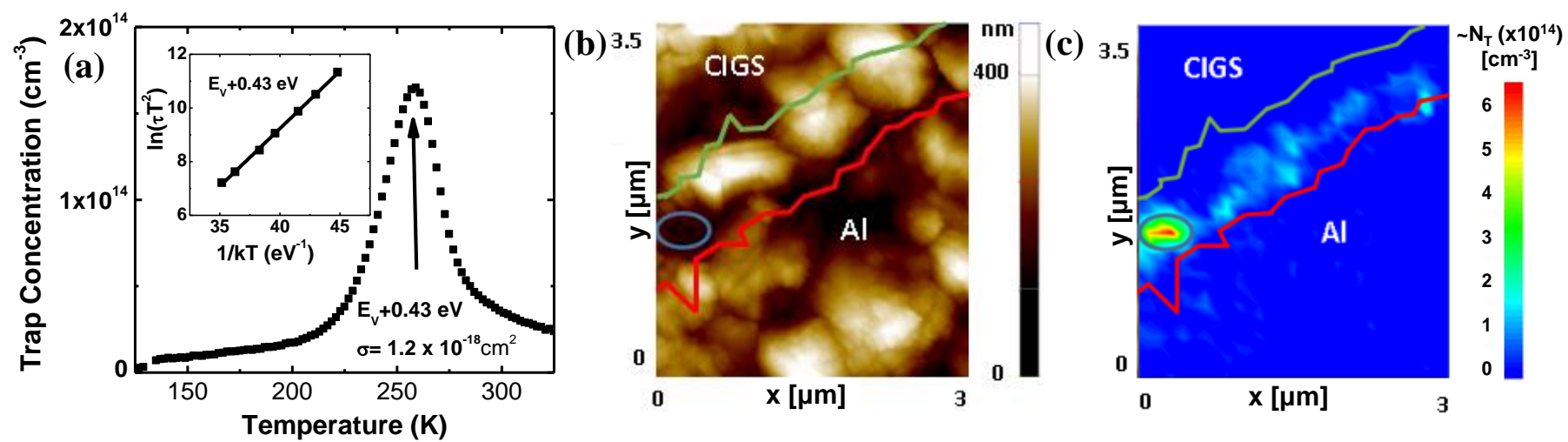

Figure 1. (a) DLTS spectrum showing a single trap level and an Arrhenius plot revealing a trap energy of $E_{V}+0.43 \mathrm{eV}$. (b) AFM image and (c) scanning-DLTS map of CIGS sample showing a region of strong localization of the $\mathrm{E}_{\mathrm{V}}+0.43 \mathrm{eV}$ trap states.

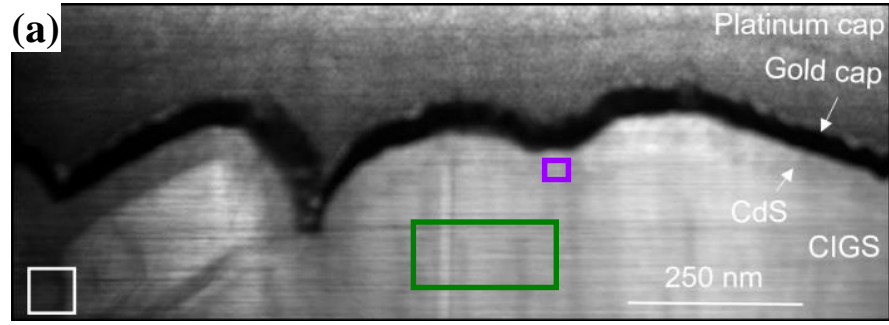

Figure 2. (a) EELS total intensity map of the CdS/CIGS specimen, with $(b, c)$ associated valence EELS spectra taken from the areas indicated in the boxes. The intergrain region within the white box (b) exhibits the $0.42 \mathrm{eV}$ peak, while regions of pure intragrain CIGS (green) and pure CdS (purple) do not (c).
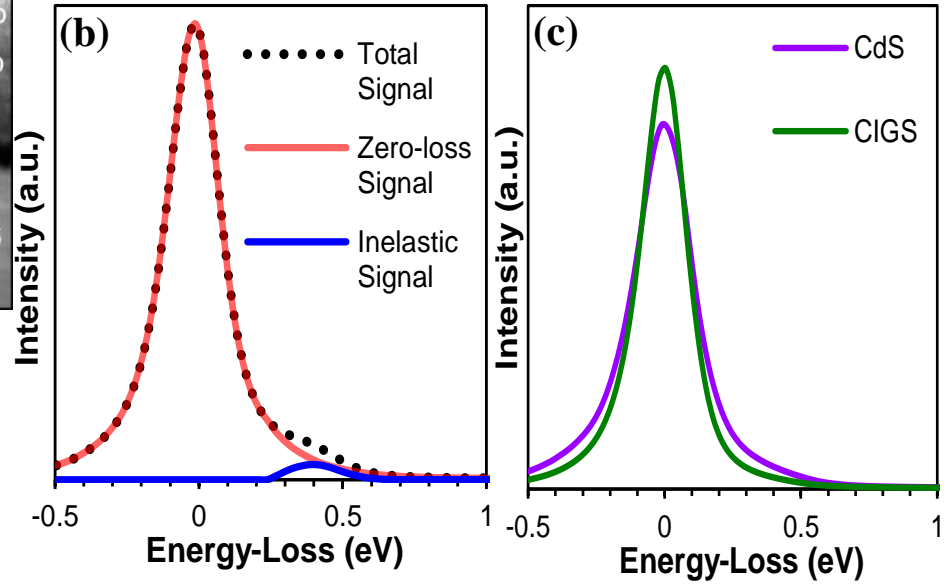